\title{
EL CAUCE BAJO DEL RÍO SANTA LUCíA. Tres propuestas basadas en sus recursos patrimoniales
}

\author{
Mercedes Medina \\ Arquitecta, Magister en Ordenamiento Territorial y Desarrollo Urbano \\ Docente e investigadora de la Facultad de Arquitectura, Universidad de la República
}

\section{RESUMEN}

En la última década se han realizado varias propuestas basadas en los recursos patrimoniales en torno al cauce bajo del río Santa Lucía en el área metropolitana de Montevideo. El origen de las iniciativas, el marco institucional en el cual se desarrollan y la gestión de las mismas es diverso, así como el éxito en la concreción de las acciones planteadas. A su vez, el concepto de recurso patrimonial, la identificación de los elementos que lo componen y su puesta en valor a través de un proyecto territorial concreto, han evolucionado de una propuesta a la otra, reflejando el estado del arte sobre esta temática en el ámbito nacional. El artículo presenta estas propuestas, realizando una descripción y evaluación de las mismas y de sus impactos y repercusiones, tanto territoriales como institucionales.

Palabras clave: recurso patrimonial, paisaje cultural, proyecto territorial, turismo

\section{ABSTRACT}

During the last ten years several proposals have been done based on natural and cultural resources in the lower course of the Santa Lucia River, in the 


\section{ID INVESTIGACIONES}

metropolitan area of Montevideo. The origin of these initiatives, the institutional frame in which they were developed, and their management are diverse, as well as the success in the concretion of the proposed actions. At the same time, the concept of heritage resource, the identification of the elements that compose it and its appreciation through a concrete land project have evolved, reflecting the state of the art on this topic in Uruguay. The article presents these proposals, carrying out a description and assessment of its land and institutional repercussions.

Key words: heritage resources, cultural landscape, land project, tourism

\section{INTRODUCCIÓN}

La puesta en valor de los recursos patrimoniales ha constituido en las últimas décadas una oportunidad para desencadenar procesos de desarrollo regional con una fuerte base local. Nuevas figuras de planificación basadas en esta premisa tienen como objetivo impulsar regiones en procesos de desarrollo, posicionándolas en contextos más amplios, basándose en la gestión de los recursos naturales y culturales y con una fuerte base territorial. A través del turismo cultural buscan el crecimiento social y económico de las localidades. En ellas los impactos negativos ocasionados por las innovaciones técnicas, las transformaciones en los modos de producción y las desterritorializaciones de las economías, pueden encontrar su contraparte en la valorización de los recursos locales. En los mejores ejemplos las huellas del pasado sobre el territorio se revalorizan involucrando en el proceso a los habitantes del lugar. La puesta en valor de este legado reafirma el sentido de pertenencia y de identidad de los habitantes y brinda la posibilidad de buscar alternativas al desarrollo local de las comunidades. En todas ellas se conjugan tres 


\section{ID_INVESTIGACIONES}

componentes: el territorio, sus recursos patrimoniales y la posibilidad de desarrollar propuestas de turismo cultural. ${ }^{1}$

Se trata entonces de tomar del propio territorio los datos en los que fundamentar su ordenación, con objeto de fortalecerlo para que sea capaz de hacer frente a los nuevos desafíos a los que está sometido constantemente, convirtiendo éstos en oportunidades para su desarrollo armónico.

Desde la Convención sobre la Protección del Patrimonio Mundial, Cultural y Natural elaborada en la $17^{\mathrm{a}}$ reunión de la Conferencia General de la Organización de las Naciones Unidas para la Educación, la Ciencia y la Cultura hasta la Carta Internacional Sobre Turismo Cultural elaborada por ICOMOS en 1999, el concepto de patrimonio se ha modificado. Se ha ampliado, incorporando entornos naturales y culturales y componentes tangibles e intangibles $y$, fundamentalmente se ha reconocido como un aspecto estrechamente ligado a la identidad de los pueblos, en el cual fundamentar el desarrollo de las comunidades. Tanto a escala internacional, nacional o local el concepto de patrimonio aparece vinculado a la interacción a lo largo del tiempo de las comunidades con el soporte físico en el que se asientan. El territorio en sí, como dimensión física y como construcción cultural, es patrimonio.

El turismo, tercer componente de las mencionadas propuestas, puede ser considerado como un fenómeno social que influye en los niveles de vida de una comunidad. Es fuente de ingresos, a la vez que provee oportunidades para la distracción, el descanso y la diversión que están directamente relacionados con el desarrollo social, económico y psicológico de personas, comunidades e

\footnotetext{
${ }^{1}$ Capítulo 4 "Precedents - Casos d'estudi europeus" realizado por Sabaté y Lista en el que se recorren 9 parques patrimoniales europeos analizando sus proyectos en función del contexto territorial y los objetivos de los mismos. En "Projectant l'eix del Llobregat. Paisatge cultural I desenvolupament regional", Universitat Politècnica de Catalunya, Massachusetts Institute of Technology. 2001.
} 


\section{ID INVESTIGACIONES}

incluso naciones (Cala Matiz, 2001). Su impacto no es sólo económico y cuando está ligado a los recursos patrimoniales, abre otras posibilidades de crecimiento social, de relación interpersonal entre el visitante y el habitante de la localidad, pudiendo convertirse en una experiencia de mutuo aprendizaje (Cóceres y otros, 2003).

En particular el turismo cultural surge del interés que tienen los visitantes por conocer la historia, la cultura y el entorno natural de las zonas que recorren. EI turismo especializado basado en la naturaleza, historia, cultura, raíces étnicas, deporte y aventura, religión, actividades económicas y profesionales, es también una modalidad en desarrollo. La incorporación de estas nuevas demandas turísticas en los manuales de la Organización Mundial del Turismo y de la Organización de las Naciones Unidas para la Ciencia y la Cultura, evidencian esta tendencia. ${ }^{2}$ En definitiva, existe una búsqueda de nuevos destinos y nuevos productos, que constituye una oportunidad para el desarrollo de las localidades.

La vinculación del turismo con el patrimonio tangible e intangible obliga a una gestión inteligente del mismo como recurso, brindando posibilidades para su interpretación. El primer paso debe ser el reconocimiento del patrimonio como capital, lo que exige además de su conservación, programas educativos y de difusión. Los programas deben construirse sobre la base de visiones integrales donde el turismo, como actividad económica y social, involucra discusiones sobre el patrimonio desde una perspectiva de desarrollo sustentable (Cóceres

\footnotetext{
${ }^{2}$ En "Gestión del Turismo en sitios del patrimonio Mundial: Manual práctico para administradores de sitios de Patrimonio Mundial", se especifican dos categorías del mercado turístico complementarias al llamado turismo tradicional: los viajeros independientes quienes buscan un entorno nuevo y diferente y el turismo especializado (en el que se incluye el turismo cultural) que busca realizar actividades específicas como deporte, investigación u observación. Especifica además varias publicaciones destinadas a este tipo de deporte, investigación u observación. Especifica además varias publicaciones destinadas a este tipo d
turista.

El "Barómetro OMT del Turismo Mundial", de 2010 vincula el turismo responsable al desarrollo local.
} 


\section{ID_INVESTIGACIONES}

y otros, 2003). La mirada desde la actividad turística permite focalizar a qué tipo de demanda interesa responder y si es posible acceder a ella, sus intereses y requerimientos específicos para determinar el producto turístico que debe conformarse. La aproximación al territorio reconociendo sus múltiples lecturas (soporte físico y funcional, construcción histórica y colectiva) permite determinar si cuenta con los recursos suficientes para conformar los productos requeridos así como las condiciones en que éstos pueden desarrollarse. Finalmente la disciplina de la ordenación territorial brinda instrumentos para la gestión de los recursos que permiten encauzar propuestas de desarrollo armónico, en una visión inclusiva de los procesos que se dan sobre el territorio, incorporando diversas escalas.

En Uruguay esta vinculación estrecha entre territorio, patrimonio y desarrollo social, económico y cultural de las localidades marca una senda que se ha comenzado a transitar recientemente. En este proceso han sido fundamentales los intercambios académicos realizados con universidades de ámbito regional y europeo. En particular el Proyecto ALFA, Red de Gestión de Recursos Culturales como Fundamento de Planes de Desarrollo Local en el que participaron las Universidades Politécnica de Cataluña, Técnica de Lisboa, Nacional de Córdoba, de Buenos Aires y Pontificia Universidad Católica de Chile durante los años 2003 al 2006 constituye un importante avance en esta dirección. En esta etapa se realizan una serie de seminarios de intercambio de experiencias llevadas adelante en los distintos países participantes, se realizan trabajos conjuntos y cursos de posgrado, sentando las bases para la profundización en esta línea de trabajo y la consolidación de la formación de técnicos e investigadores en la temática.

En el ámbito territorial uruguayo varias áreas reúnen las características necesarias para desarrollar propuestas basadas en sus recursos patrimoniales naturales y culturales como alternativa o complemento a la tradicional oferta de 
ID_INVESTIGACIONES

sol y playa de la costa Sur, o del turismo termal del litoral Oeste. En particular el entorno del río Santa Lucía, en el área metropolitana de Montevideo, es una de ellas. Desde finales de los 90 se han desarrollado varias propuestas sobre la misma, todas ellas basadas en los recursos patrimoniales y apuntando a su

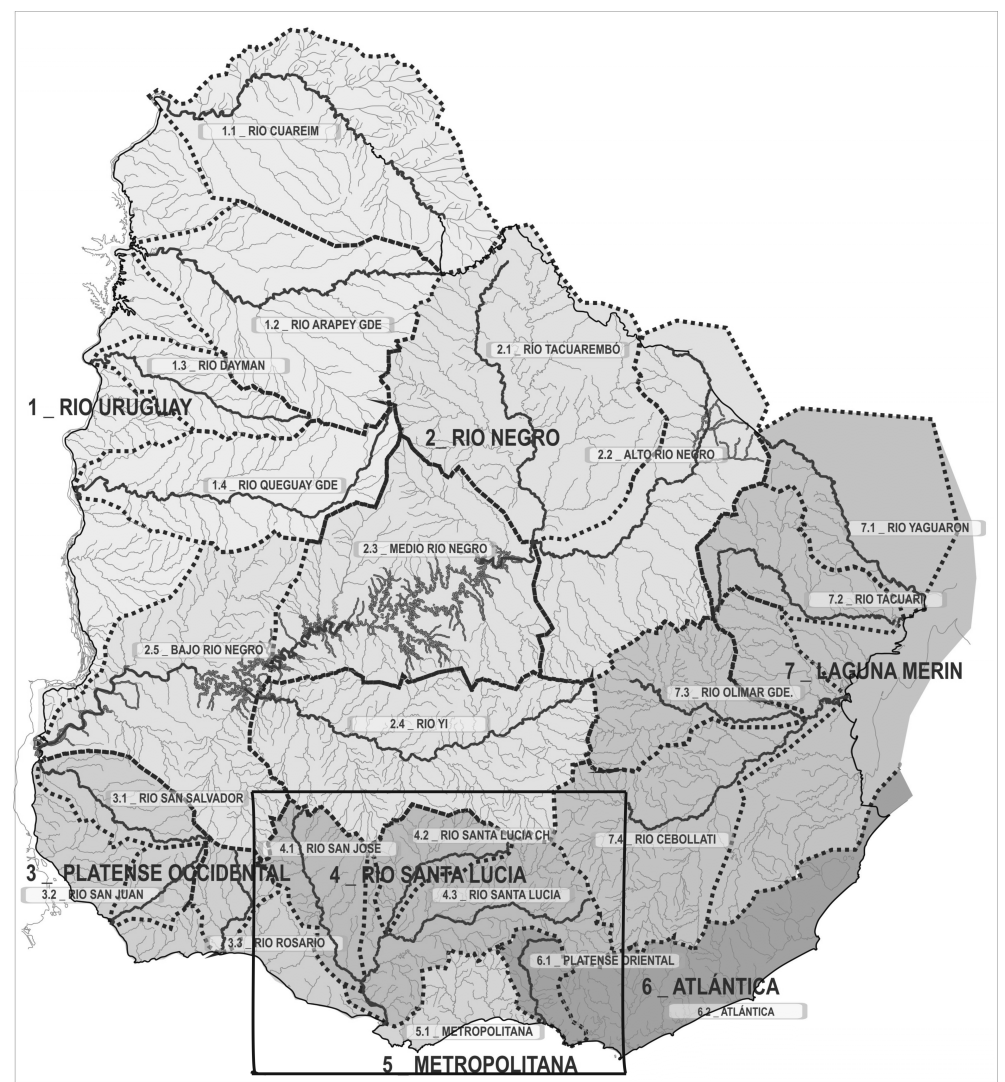

Principales cuencas hídricas del Uruguay

Fuente: Mesa de Recursos Hídricos UdelaR 2007 


\section{ID_INVESTIGACIONES}

puesta en valor a través del turismo cultural. Por medio de estas propuestas es posible recorrer el camino que ha experimentado la consideración del tema patrimonial desde la perspectiva de la construcción de un proyecto territorial, así como evaluar las repercusiones que ha tenido cada una, permitiendo formular algunas conclusiones que aporten en la concreción de esta manera de operar. Se presentarán tres de estas iniciativas. En una primera instancia se explicará su origen, el marco institucional en el que se desarrollan y el marco conceptual aplicado en cada una. Se realizará una breve descripción de la propuesta territorial, procurando explicitar los aspectos necesarios para su comprensión. En las consideraciones finales se evaluarán y compararán los impactos y resultados obtenidos en los tres casos.

\section{EL TERRITORIO DEL RÍO SANTA LUCÍA}

El río Santa Lucía, con una longitud de 225 kilómetros, define una de las cuencas hidrográficas más importantes del Uruguay. En ella se localiza gran parte de la producción granjera y hortifrutícola del país. El río abastece de agua potable a la ciudad de Montevideo y a gran parte de la región metropolitana que concentra el $60 \%$ de la población total del país. La localidad de Aguas Corrientes, 47 kilómetros aguas arriba de la desembocadura del Santa Lucía en el Río de la Plata, debe su nombre a la actividad que le dio origen y que aún hoy desarrolla. Junto con las modernas instalaciones de la compañía de aguas del estado se conserva la primera planta potabilizadora inglesa de comienzos del siglo XIX.

Es el curso de agua interior más importante de la zona Sur del Uruguay. Su último tramo se desarrolla totalmente dentro del área metropolitana de Montevideo, delimitando los tres departamentos que la conforman: Montevideo, Canelones y San José. Por la amplitud y profundidad de su cauce constituyó un límite también para la localización de los primeros pobladores del territorio

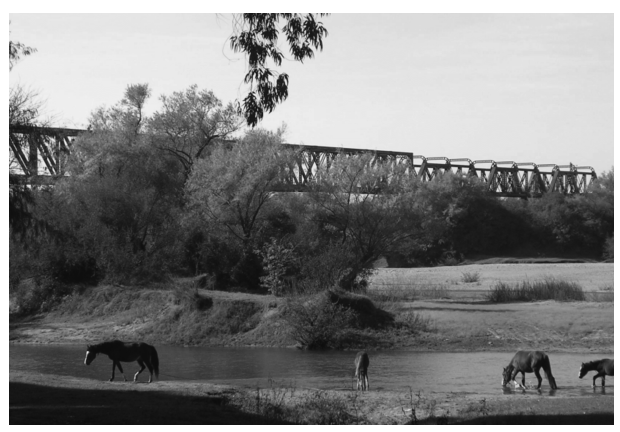

Río Santa Lucía, monte nativo y arenales 


\section{ID_INVESTIGACIONES}

metropolitano. En la margen Este se concentra el uso intensivo de chacras, mientras que al Oeste los predios son mayores, teniendo su origen en las primeras estancias en las que se criaba ganado y que hoy dan asiento a parte de la cuenca lechera Sur. Ambas situaciones definen paisajes productivos estrechamente vinculados a los primeros pobladores, inmigrantes europeos, que llegaron al Uruguay a partir de los primeros planes de colonización del siglo XVIII. En muchos casos es posible reconocer las trazas viales sobre las que se desarrollaron estos primeros fraccionamientos rurales.

Su curso inferior está influenciado por la marea eólica que ingresa desde el Río de la Plata provocando una intrusión salina que genera un ecosistema único sobre 20.000 hectáreas en las que se forma un extenso humedal. Este particular ecosistema, único en la costa platense uruguaya, se localiza a 13 kilómetros de la ciudad de Montevideo y se desarrolla en los tres departamentos del área metropolitana. El paisaje natural se destaca por su belleza panorámica en la que el bosque indígena fluvial se intercala con la extensión del humedal. La proximidad a la ciudad capital y los valores paisajísticos de la zona hicieron del cauce bajo del Santa Lucía, un área preferencial para la localización de las residencias de veraneo de las clases altas de la sociedad montevideana del siglo XIX. Muchas de estas residencias se conservan constituyendo parte del patrimonio del área, tanto por su arquitectura, como por los personajes que las habitaron y en muchos casos, por el diseño de sus jardines.

Finalmente debe mencionarse el patrimonio arqueológico, que evidencia ocupaciones humanas prehistóricas, así como la vinculación del río a varias instancias relevantes de nuestra historia, ya que constituyó una barrera natural de defensa de la ciudad de Montevideo al oeste de la misma.

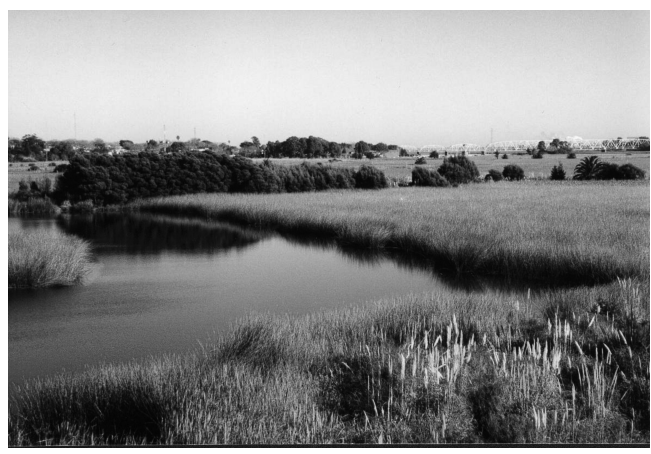

Humedales del río Santa Lucía 


\section{ID_INVESTIGACIONES}

Si bien la intervención humana manifestada a través del trazado de infraestructuras viales de acceso a la capital, poblados de diversas características, que en general presentan problemas ambientales y niveles socioeconómicos bajos, localización industrial y extracción de arenas, ha provocado alteraciones en el ecosistema y el patrimonio arqueológico, es posible encontrar vastas áreas con bajo grado de alteración y en las que se conserva la flora y fauna originales. De esta manera, se posiciona como un área de destacados valores paisajísticos, ambientales y patrimoniales que, con la adopción de medidas orientadas hacia su protección, puede ser accesible para que un importante número de visitantes puedan conocerla y disfrutarla, así como para la implementación de programas de educación ambiental y fundamentalmente, para incorporar posibilidades de desarrollo a las comunidades locales.

Así lo ha entendido el Proyecto $\mathrm{SNAP}^{3}$ ya que está previsto su ingreso al Sistema Nacional de Áreas Protegidas estando en elaboración su plan de manejo. Esta propuesta se centra en las características ecosistémicas en torno al territorio del humedal y a la desembocadura del río y se basa en el reconocimiento de la diversidad de ambientes que constituyen hábitat para numerosas especies vegetales y animales, además de sus potencialidades para el desarrollo de actividades recreativas y deportivas.

\footnotetext{
3 "El Proyecto SNAP es un apoyo para la puesta en marcha del Sistema Nacional de Áreas Protegidas (SNAP) que, liderado por la Dirección Nacional de Medio Ambiente (DINAMA), reúne aportes del Gobierno nacional, gobiernos departamentales, otras instituciones públicas y organizaciones privadas y sociales, y contribuciones de la cooperación internacional multilateral (Programa de las Naciones Unidas para el Desarrollo y Fondo para el Medio Ambiente Mundial) y bilateral (España y Francia). El tiene por objetivo apoyar el diseño e implementación inicial de un SNAP que conserve efectivamente una muestra representativa de la biodiversidad del pais. En este sentido, articula componentes de diseño y planificación, que implican definiciones a aplicar y ejecutar durante su horizonte temporal (cinco años) y más allá del mismo". www.snap.gub.uy,
} 


\section{ID_INVESTIGACIONES}

A continuación se presentan tres propuestas basadas en los recursos naturales y culturales que abarcan la totalidad del área o parte de ella. Han surgido desde diferentes ámbitos y tienen diferente grado de aceptación y concreción.

\section{AMPLIACIÓN ÁREA SUROESTE. Proyecto integrado de Turismo, Patrimonio y Ambiente. Programa Turístico}

En el año 2000 se firma un convenio entre tres gobiernos departamentales del Suroeste uruguayo y el Ministerio de Vivienda, Ordenamiento Territorial y Medio Ambiente, para la elaboración de un programa integrado de "Patrimonio, Turismo y Ambiente" en la región, conformada por los departamentos de San José, Colonia y Soriano. Este programa, de escala regional, tuvo como objetivo lograr la protección y desarrollo durable de sitios naturales y patrimoniales para el Área Suroeste (proyecto ASO, 1997), dotándolos de un sentido económico a través del turismo. Dos de los tres departamentos involucrados en la propuesta se caracterizan por una importante producción lechera y de granja. Fundamentalmente el departamento de Colonia incluye varias localidades cuyo origen ha sido la inmigración valdense que ha trasladado y adaptado a nuestro territorio los modos de producir y las costumbres de sus tierras de origen, caracterizando el área. Sobre el Río de la Plata y frente a la ciudad de Buenos Aires, Colonia del Sacramento, capital de éste departamento, es la primera fundación en nuestro territorio en 1680 y la única de origen portugués. Actualmente está designada como Patrimonio Mundial de la Humanidad por la Unesco, lo que constituye un centro de atracción para toda la región. La industria extractiva, fundamentalmente de arena en la costa, abasteció a la ciudad de Buenos Aires en el siglo pasado y ha dejado un par de localidades que albergaban infraestructura y trabajadores 
ID_INVESTIGACIONES

vinculados a esta actividad. En el departamento de Soriano, sobre el río Uruguay, el panorama cambia. Este departamento se caracteriza por un desarrollo productivo ganadero-cerealero. Su principal atractivo son los ambientes naturales, fundamentalmente vinculados a los cursos de agua principales y el patrimonio arqueológico histórico y prehistórico.

La propuesta formulada para esta extensa región se basa en dos aspectos fundamentales: el concepto de patrimonio con el que se trabaja, y la considera-

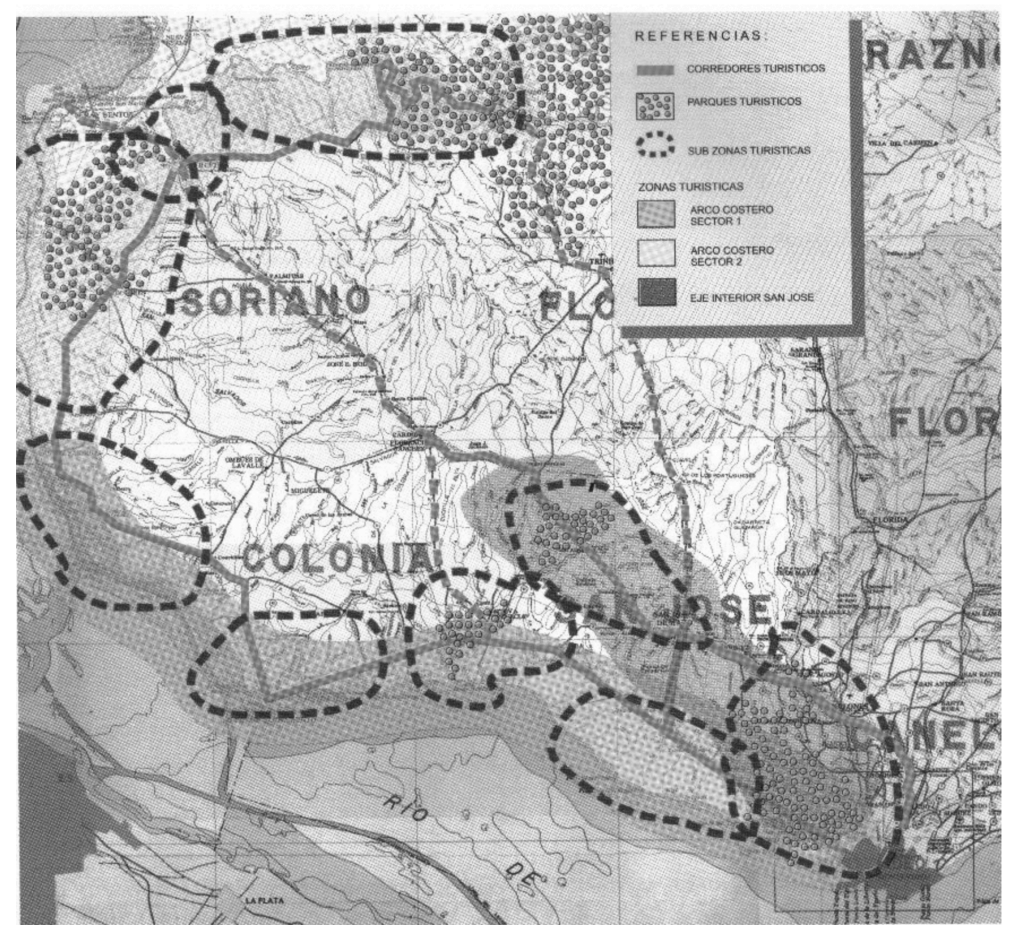

Zonas y parques turísticos. Fuente: Ampliación ASO. Programa de Turismo, Patrimonio y Ambiente MVOTMA/DINOT/IMC/IMSJ/IMS 2000 
ción del desarrollo económico como una herramienta para su defensa y fortalecimiento.

Con relación al primer aspecto se considera el patrimonio conformado por bienes naturales y culturales y por sus entornos. Incorpora asimismo la consideración del patrimonio de escala local. Ambos aspectos no estaban incluidos en la Ley de Patrimonio Histórico, Artístico y Cultural de la Nación, vigente desde 1971. Si bien existieron algunos avances desde la década del 80 en los que se reconocen, además del significado histórico, la calidad arquitectónica, la urbana y la ambiental, estos se reflejaron en propuestas de gestión en entornos exclusivamente urbanos en la ciudad de Montevideo. La propuesta del Área Suroeste constituye un avance en relación a la inclusión de estos temas en figuras de planificación.

A su vez se trabajó con el convencimiento de que la formulación de una propuesta turística basada en el patrimonio del área y su interpretación, garantizando las calidades ambientales, permitiría conservar este patrimonio fortaleciéndolo, al convertirlo en protagonista del desarrollo. La apuesta fundamental de este programa de "Patrimonio, Turismo y Ambiente" se centra en el desarrollo de un plan turístico de escala regional, con respaldo territorial y patrimonial. La implementación de una gestión integrada que permitiera prever, evaluar y mitigar los posibles impactos negativos que las actividades turísticas pudieran tener sobre el territorio tornaría la región más atractiva para los inversores. El programa desarrolla propuestas empresariales y territoriales. Las primeras incluyen programas de animación y la posibilidad de conformación de redes o grupos de empresarios turísticos, las segundas se concretan en un proyecto territorial para los tres departamentos del Área Suroeste.

El proyecto territorial define tres zonas turísticas diferenciadas entre sí y estructuradas y vinculadas por corredores viales. Para su determinación se tuvo en cuenta la existencia de recursos turísticos y patrimoniales, sus poten-

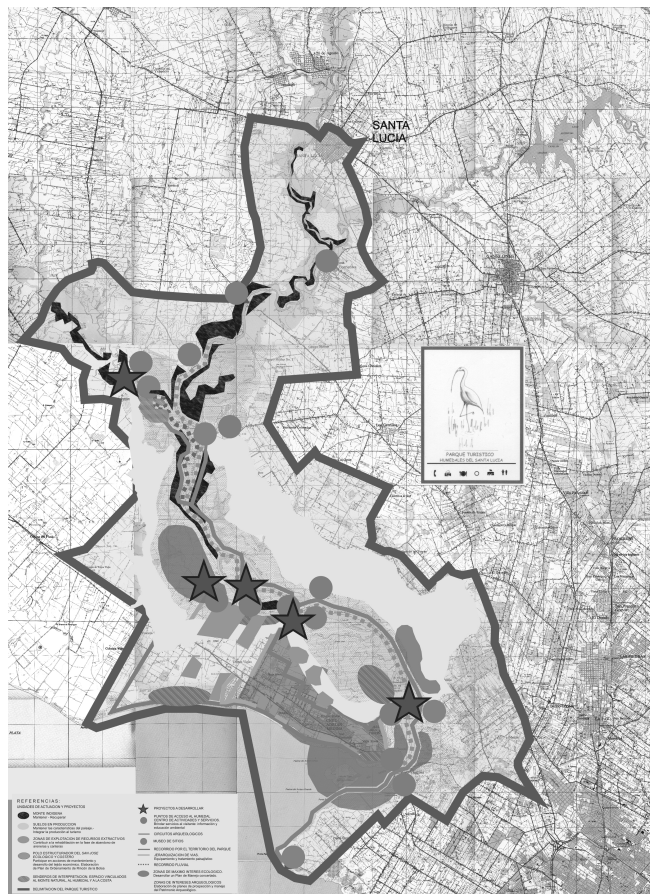

Propuesta Parque Turístico del Santa Lucía. Fuente: Ampliación ASO. Programa de Turismo, Patrimonio y Ambiente MVOTMA/DINOT/IMC/IMSJ/IMS 2000 


\section{ID_INVESTIGACIONES}

cialidades y su estado de desarrollo. Estas zonas son: el arco costero del Río de la Plata, el arco fluvial del Río Uruguay, y corredor interior a través del territorio productivo. A su vez se identifican áreas urbanas y su entorno, desarrollos costeros y áreas de concentración de recursos patrimoniales naturales y culturales específicos que conforman a su vez subespacios que pueden ser caracterizados como una unidad. Es en estos que se desarrolla la propuesta de cinco parques turísticos, uno de ellos en el cauce bajo del río Santa Lucía. Los límites administrativos departamentales se diluyen en una apuesta integradora de la región.

La figura del parque turístico se basa en dos componentes fundamentales: el territorio en el que se desarrolla y una organización aplicada a este espacio que garantiza la gestión integrada del ambiente, la economía, el turismo y la ordenación del territorio. La finalidad del Parque Turístico es, por tanto, encauzar su territorio en un proceso de desarrollo apoyado en la puesta en valor de sus recursos patrimoniales, incorporándole productos turísticos, y basado en la sustentabilidad de esos recursos. (Ampliación ASO, 2000)

Se formulan objetivos específicos: desarrollo económico, formulación de un proyecto de territorio, involucramiento de la población y monitoreo de los procesos sobre ese territorio.

En el proyecto para el Parque Turístico del Santa Lucía participan arqueólogos, especialistas en flora y fauna, agrónomos, expertos en patrimonio y en ordenación territorial. Con ellos se caracteriza y delimita el ámbito territorial de actuación que se describe en una Carta patrimonial y natural. Se detectan y describen, a modo de inventario, los recursos más relevantes: monte nativo, pradera natural, comunidades psamófilas, se caracteriza el humedal y se describe la fauna y flora y los recursos arqueológicos. También se detectan y describen las amenazas: actividades extractivas, urbanizaciones en áreas con exceso de humedad por el tipo de suelo que impide su drenaje, industrias. Con 


\section{ID INVESTIGACIONES}

estos insumos se formula un proyecto territorial específico sobre una superficie de 60.000 hectáreas que abarca el ecosistema del humedal y un área de amortiguación de impactos sobre el mismo. Se localizan puertas de entrada al área asociadas a las rutas principales de acceso ${ }^{4}$, centros de visitantes, museos de sitio, circuitos fluviales y senderos de interpretación por los diferentes ecosistemas, áreas de acceso restringido, y distintos niveles de uso turístico.

Paralelamente se establece un programa de acciones que deben llevarse a cabo en el ámbito del parque para la consecución de los objetivos citados anteriormente. Dichas acciones están dirigidas a apoyar la gestión de los productores asociados, a fomentar programas de educación ambiental e investigación, a promover y a garantizar la calidad de los productos ofrecidos a través de una marca particular que los asocia con el territorio. En síntesis, busca apoyar el proceso de desarrollo y a asegurar la calidad y sustentabilidad del mismo.

El Parque se instrumenta a través de Programas de Desarrollo Local a cargo de los gobiernos municipales, con apoyo del gobierno nacional en primera instancia. Este apoyo consistiría en la obligatoriedad de evaluación de impacto ambiental a todos los emprendimientos propuestos en el área y en la declaración de interés turístico nacional con el fin de favorecer las iniciativas en este sentido. Los organismos nacionales directamente involucrados son los Ministerios de Vivienda, Ordenamiento Territorial y Medio Ambiente y el de Turismo ${ }^{5}$. Finalmente el Parque es gestionado por una Sociedad de Economía ${ }^{4}$ Estas puertas de entrada se definen como elementos que oficien de mojones y que
ubiquen al turista en dichas áreas. (Ampliación ASO, 2000)
5 En el momento en que se realiza esta propuesta, aún no estaba aprobada la Ley de Ordenamiento
Territorial y Desarrollo Sostenible que establece procedimientos aplicables en este tipo de propuestas. La Tersma fue sancionada recién en 


\section{ID INVESTIGACIONES}

Mixta, de carácter regional, construida durante el proceso de conformación del proyecto. En ella deben estar representados todos los actores del parque, manteniendo el $51 \%$ de las acciones en manos de las instituciones públicas.

En el año 2000 esta propuesta es presentada públicamente en las capitales de los departamentos y entregada a las autoridades de las instituciones nacionales y departamentales, sin llegar a concretarse.

\section{EL RÍO SANTA LUCÍA, UNA OPORTUNIDAD DE FORTALECER EL} TERRITORIO

En el año 2005 se desarrolla una segunda propuesta para el tramo inferior del río Santa Lucía. En este caso desde el ámbito académico dentro de la investigación "Construir el futuro revalorizando el pasado. El turismo cultural, una oportunidad de desarrollo en el territorio metropolitano de Montevideo", se retoma el ámbito territorial del bajo Santa Lucía en el que se profundiza en un proyecto de parque patrimonial. La investigación es desarrollada en el marco conceptual del mencionado Proyecto ALFA y se propone vincular la construcción cultural del territorio metropolitano de Montevideo y su potencialidad como recurso y factor de desarrollo turístico.

Las particularidades del territorio metropolitano de Montevideo se presentan como un desafío que debe abordarse a corto plazo. Sobre una matriz territorial heredada conformada por el soporte físico y las formas de uso y ocupación tradicionales de la producción agropecuaria, se superponen nuevas funciones y modos de ocupación. La localización de grandes equipamientos, de la infraestructura logística y de transporte y las nuevas pautas de consumo como las urbanizaciones cerradas y las grandes superficies comerciales, coexisten con la extensión informal del área urbanizada, generando zonas de conflicto con la producción tradicional. 


\section{ID_INVESTIGACIONES}

La interacción entre naturaleza y cultura adquiere expresiones específicas. El reconocimiento de estas especificidades justifica una mirada diferente sobre el territorio, de la que pueda extraerse una reinterpretación del mismo en la cual apoyar una opción de desarrollo a través de un turismo sustentable. Aun considerando que ésta no es la única posibilidad de desarrollo, ni aún una opción posible en muchos casos, ya que son muchas las variables que deben confluir para la conformación de un producto turístico, se plantea que, a partir del reconocimiento del propio territorio podrán extraerse la claves para su puesta en valor.

Uno de los desafíos que se presentan en la investigación es la identificación de los elementos que permiten describir este territorio de manera holística, incorporando las múltiples dimensiones que lo conforman. Se seleccionan tres variables en las que basar una alternativa de desarrollo. La primera de ellas es la construcción histórica del territorio. Una serie de mapas explican cómo se ha ido ocupando a lo largo del tiempo y detecta en cada etapa las huellas que han permanecido en la estructura territorial.

La segunda es el paisaje como elemento de síntesis de las formas de uso y ocupación del suelo, resultado de la interacción entre naturaleza y cultura. El producto en este caso es la identificación de trece unidades de paisajes diferenciadas y delimitadas considerando las texturas sobre el territorio que conforman el parcelario, la estructura de los caminos, el uso del suelo, las características de localización de la población dispersa o en enclaves urbanos más o menos próximos, y las características geomorfológicas. A esta lectura se superponen las áreas con valores ecológicos relevantes que, tanto por su interés, como por su fragilidad, merecen atención especial en el contexto metropolitano.

La tercera son las actividades productivas tradicionales en las que pueden apoyarse o potenciarse alternativas para su uso como espacio de recreación.

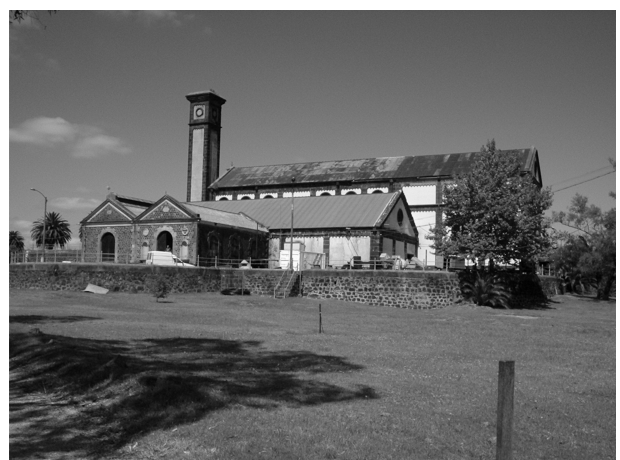

Primera planta potabilizadora del S XIX en Aguas Corrientes 


\section{ID_INVESTIGACIONES}

En particular el territorio metropolitano de Montevideo se caracteriza por unidades productivas de menos de 5 hectáreas que tradicionalmente son explotadas por pequeños productores que viven en ellas con sus familias. La consideración del patrimonio intangible se incorpora a través de la identificación de fiestas y eventos de diverso origen y alcance. Algunas de estas festividades, fundamentalmente las religiosas, provienen de los primeros inmigrantes que llegaron a nuestras tierras, generalmente procedentes de España o Italia en el entorno de Montevideo; otras son de creación más reciente y están vinculadas a eventos deportivos, actividades productivas, algunas tradiciones $\mathrm{o}$ simplemente con fines recreativos. Muchas convocan a los habitantes de la localidad que la realiza, pero otras tantas tienen alcance nacional o internacional.

La territorialización de estas variables permite la detección de áreas de concentración de recursos y una aproximación a la delimitación del territorio metropolitano como espacio de recreación y turismo. Se conforma así un potencial Atlas Turístico del territorio metropolitano de Montevideo, del que forma parte el tramo inferior del río Santa Lucía. El río Santa Lucía concentra en el recorrido de su cauce principal diversos atractivos: nace y desemboca en áreas de interés ecológico relevante; concentra áreas de potencial arqueológico, las que fundamentalmente en su desembocadura son objeto de investigación desde hace algunos años; atraviesa todos los paisajes del territorio metropolitano; vincula centros poblados cuyas fundaciones responden a distintos momentos de la conformación del territorio. Ha estado vinculado a diversos hechos históricos desde las primeras llegadas de los navegantes a estas tierras y ha determinado, como barrera natural, la temprana colonización del los territorios metropolitanos del Este. (Medina, 2005)

Con la propuesta anteriormente descrita para el Parque Turístico del Santa Lucía como antecedente e incorporando la consideración de las variables 
ID_INVESTIGACIONES

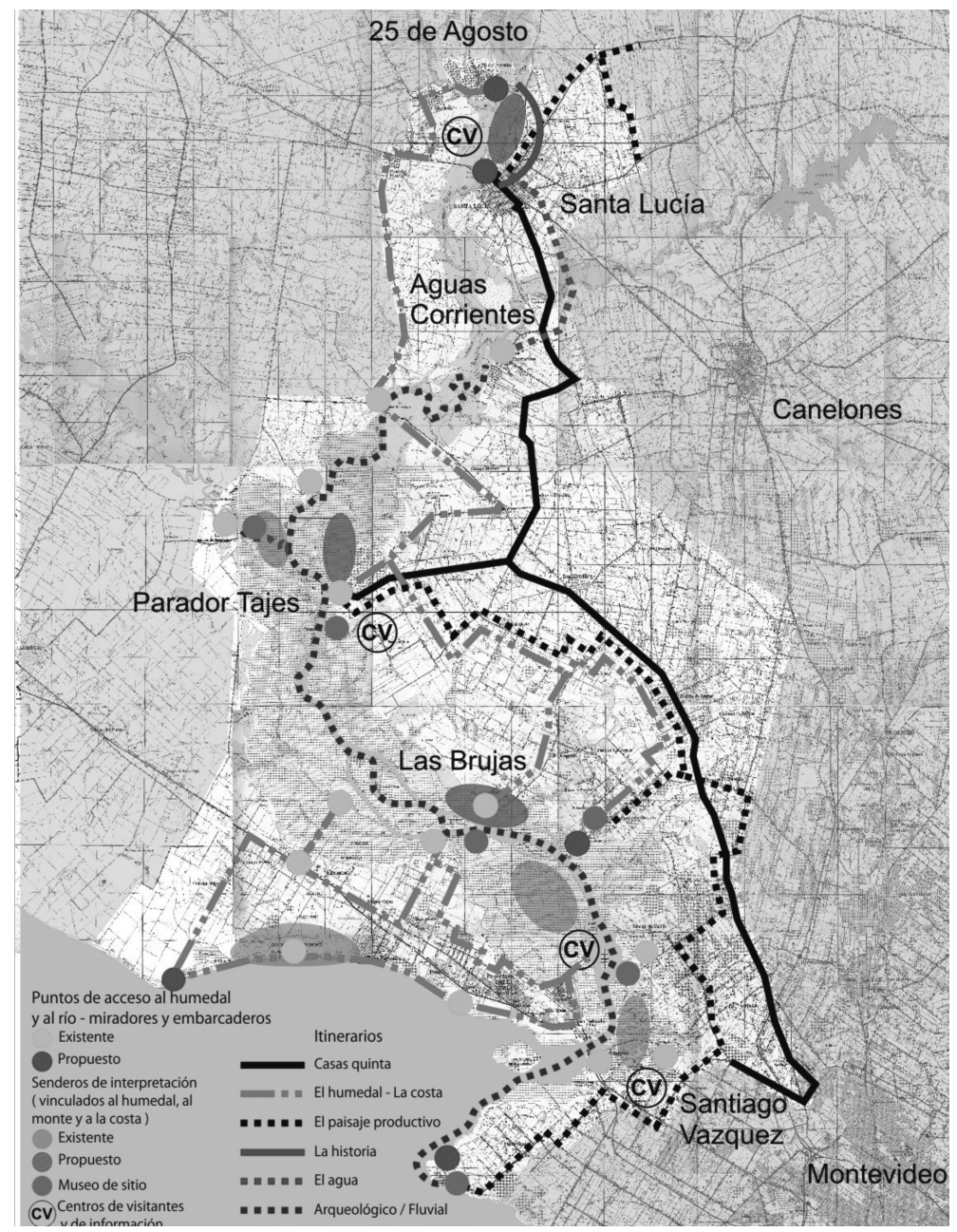

El río Santa Lucía, una oportunidad de fortalecer el territorio

Estructura del parque, recursos e itinerarios propuestos. Fuente: Construir el futuro revalorizando el pasado. M.Medina. 2005 


\section{ID INVESTIGACIONES}

mencionadas se realiza un nuevo avance para un proyecto territorial en torno a esta área.

Este adopta la figura de parque patrimonial cuya propuesta concreta se basa en los cinco puntos de análisis planteados en el estudio de antecedentes para la propuesta sobre el Río Llobregat ${ }^{6}$ : importancia del ámbito considerado; temas y objetivos de la intervención; inventario selectivo de los recursos del área; proceso de planeamiento y desarrollo que implica la gestión y la estructura de la propuesta apoyada en los conceptos e instrumentos específicos para el desarrollo de un parque patrimonial.

La identificación de nuevos recursos en esta investigación permite proponer tres itinerarios temáticos principales, basados en los recursos más relevantes del área:

1. La transición histórica que, a través del recorrido por las casasquintas, residencias de veraneo de la clase acaudalada montevideana, establece una narración que explica el modo de vida de la sociedad de finales del siglo XVIII y principios del XIX y algunos eventos históricos asociados al surgimiento de la nación y su consolidación como estado independiente.

2. La aproximación y conocimiento del ecosistema que, apoyado en la caminería existente, busca los posibles puntos de contacto con el río y el humedal.

3. En tercer lugar se plantea un itinerario a través de los establecimientos productivos, que permite tomar contacto con las familias de productores que han construido este paisaje a través de varias generaciones.

${ }^{6}$ Op. Cit 1. 


\section{ID INVESTIGACIONES}

Cuatro itinerarios secundarios, derivados de los anteriores, recogen otras miradas de interés:

- Un pequeño itinerario que conecte las antiguas casas de veraneo de la burguesía montevideana de la segunda mitad del siglo XIX, con la fortificación construida para defender el puente ferroviario de la localidad de 25 de Agosto de los enfrentamientos entre caudillos (líderes políticos), enfrentados en ese mismo período. El monte nativo y el río fueron protagonistas en ambos casos, como marco natural para las casas de veraneo y como refugio para los combatientes.

- El camino del agua, entre la localización de la primera vertiente de agua mineral del país que fue explotada comercialmente y la primera planta potabilizadora para el abastecimiento de Montevideo y su área metropolitana, ambos del S XIX.

- Un recorrido por los museos de sitio detectados en el informe arqueológico del proyecto Área Suroeste que permite acercarse a los pobladores originales.

- La navegación del río donde, a pesar de la existencia de pequeños saltos de agua, éstos no son obstáculo para que exista actividad náutica en diversas modalidades. Se trata de potenciar este aspecto asociado al deporte y a la pesca.

En cada caso se detectan temas periféricos, puntos de interés complementarios al central y la posibilidad de articularse unos a otros.

Las tres narraciones desarrolladas en los itinerarios principales propuestos ya han supuesto incipientes procesos de puesta en valor con distinto grado de 


\section{ID INVESTIGACIONES}

avance, por lo que están incorporadas en el imaginario de sus habitantes. Estos procesos, a su escala, articulan intereses privados y públicos.

Estas tres lecturas brindan la posibilidad de vincularse permanentemente a través de referencias mutuas. La presencia del río promovió el primer enclave turístico del país, los montes ribereños y los pasos en el río fueron protagonistas en el período histórico que se propone poner en valor. Las características del suelo permitieron el asiento de los primeros productores rurales.

Finalmente es posible articular esta propuesta con otras que puedan surgir en el territorio metropolitano. Este aspecto se desarrolla en el trabajo que se presenta a continuación.

\section{DESARROLLO DE LÍNEAS ESTRATÉGICAS PARA LA PROMOCIÓN TURÍSTICA DE ESTABLECIMIENTOS AGROPRODUCTIVOS EN EL OESTE DE CANELONES}

Esta propuesta se desarrolla en el año 2008, a iniciativa del gobierno departamental de Canelones. Abarca el Oeste del departamento, parcialmente incluido en las dos propuestas anteriores. Esta zona está estrechamente vinculada al río Santa Lucía.

El objetivo del trabajo, es la detección de establecimientos vinculados al agro con potencial turístico, propiciando líneas estratégicas para la promoción de agroturismo.

Se parte de tres insumos básicos: un listado de operadores y de productores interesados en desarrollar propuestas de este tipo en el territorio, un listado de grupos organizados que están trabajando en el área y una serie de 


\section{ID_INVESTIGACIONES}

antecedentes (reflexiones y propuestas sobre el territorio metropolitano), entre los que se cuentan los descritos anteriormente.

El abordaje se realiza desde las siguientes aproximaciones: un desarrollo teórico conceptual en el que se explicitan las posturas sobre el turismo y el territorio; el estudio de las propuestas procedentes de diversos ámbitos para la zona y por último una serie de trabajos de campo. Estos últimos implican recorridos por el territorio, consultas a diversos actores y reuniones con productores de la zona, que son los principales destinatarios y protagonistas de la propuesta final. El proyecto se construyó junto con los actores en el territorio. Se trabaja en contacto con el gobierno departamental que lleva adelante el programa que permite desarrollar la propuesta.

Nuevamente este trabajo vincula turismo con soporte territorial y desarrollo local.

Si bien la propuesta debe centrarse en el turismo agroproductivo, se propone ampliar esta visión de enclaves productivos a la puesta en valor del paisaje natural y cultural. El proyecto territorial se construye considerando el agroturismo como parte de un patrimonio más amplio.

El traslado en el territorio y las narraciones que lo acompañan se consideran parte fundamental de la experiencia, ya que el territorio productivo se ha construido a lo largo del tiempo, iniciándose con los primeros pobladores del territorio nacional, alguna de cuyas familias aún permanece. De hecho a los habitantes del departamento de Canelones se les llama canarios en relación a la procedencia predominante de las primeras familias que se establecieron en este territorio. La producción está entonces directamente vinculada a los orígenes e identidad de sus pobladores. La descripción se enriquece con la memoria de los habitantes y sus familias, pioneras en tierras canarias.

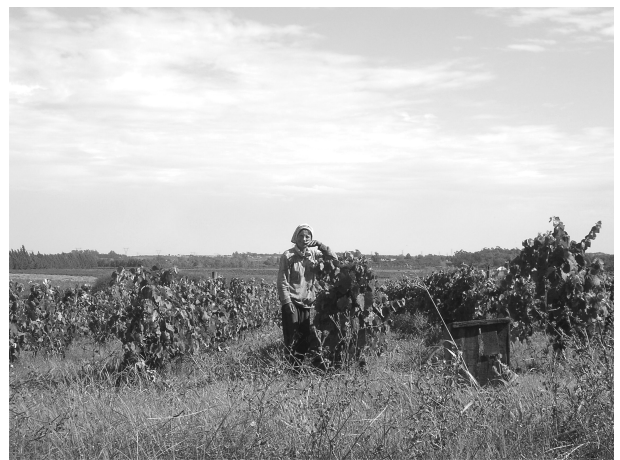

Productores rurales del área de Canelón Chico 


\section{ID_INVESTIGACIONES}

Estas consideraciones permiten fijar los criterios con los que delimitar el territorio de actuación. Se considera necesario definir un ámbito concreto y las estrategias para caracterizar su oferta, vinculándola directamente al soporte geográfico y a las comunidades que lo habitan. En este sentido se explora en la posibilidad de gestionar una marca que identifique el Oeste canario y su propuesta. Se discuten distintas posibilidades, ya que en el territorio existen iniciativas para asociar productores con una marca, superponiéndose en más de una ocasión. Otro de los temas considerados es la posible articulación de la propuesta para este territorio con el resto del departamento de Canelones, hoy fuertemente centrado en la oferta costera e incipientemente en las grandes bodegas.

Se incorporan el patrimonio natural, cultural y arquitectónico no vinculado a aspectos productivos y el patrimonio arqueológico que, junto con los usos y costumbres de los habitantes locales, permiten construir un proyecto de desarrollo turístico sustentable sobre el territorio. No obstante quedan abiertas otras posibilidades como la inclusión de las instalaciones ferroviarias de finales del siglo XIX hoy en desuso, las escuelas rurales que conforman un conjunto de notable interés histórico y, de la misma manera, las instalaciones religiosas, entre otros.

El proyecto se construye sobre un anillo vial interno, que permite recorrer el territorio al margen de las rutas nacionales que hoy lo atraviesan. Sobre estas rutas nacionales y en las capitales departamentales como potenciales emisoras de turistas, se plantea la localización de puertas de acceso a la oferta de puesta en valor de este territorio productivo.

Se identifican áreas de concentración de recursos y cinco proyectos especiales, de valorización de elementos singulares: un viejo puente sobre el río Santa Lucía Chico en una zona de escenarios relevantes, el embalse de una represa, un par de pequeñas localidades en las orillas del río, la 
ID_INVESTIGACIONES

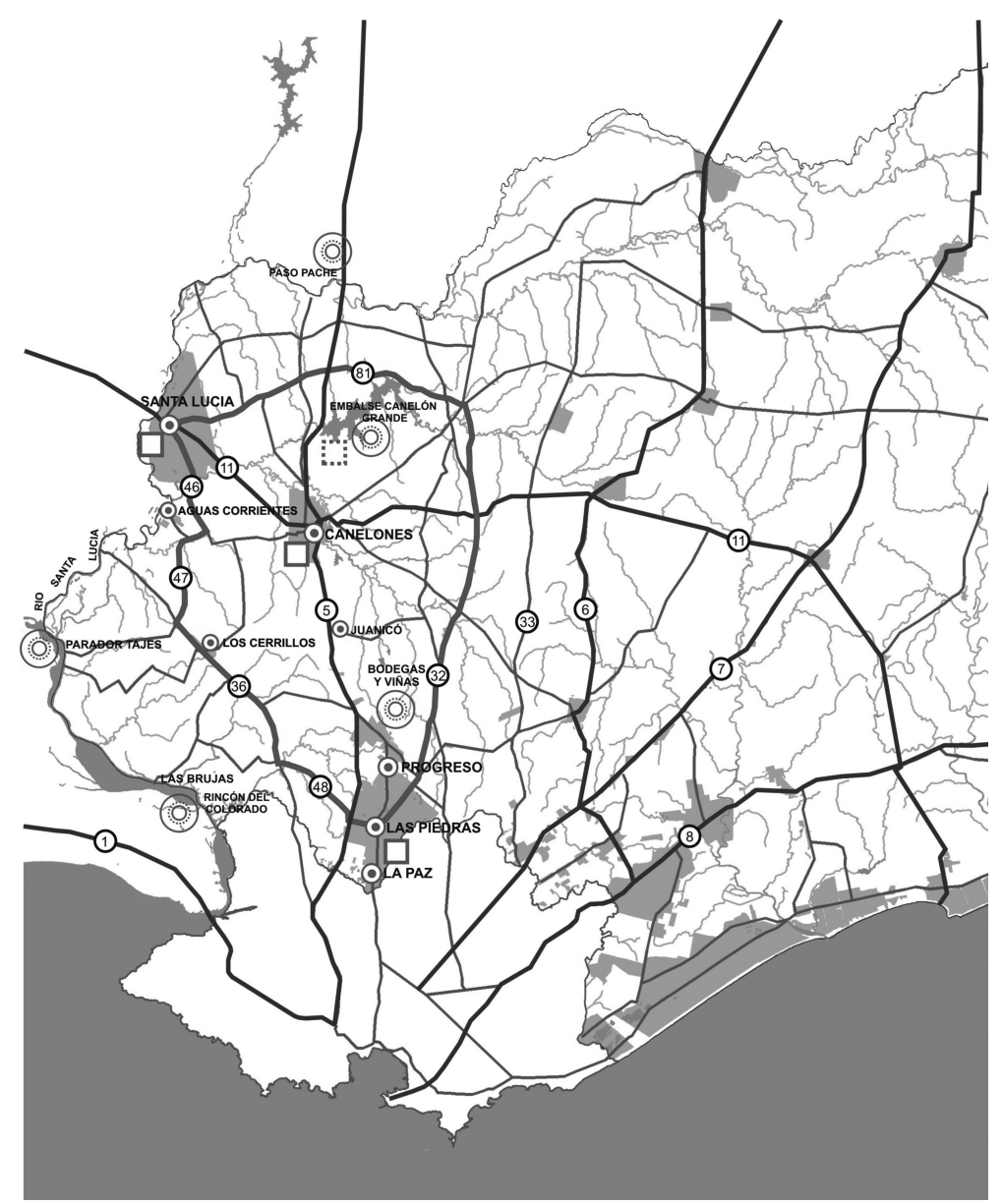

Estructura territorial propuesta

Anillo vial interno, portales de entrada, áreas de concentración de recursos y proyectos especiales.

Fuente: Turismo agroproductivo en el oeste canario. CEEC/Comuna Canaria 2008 


\section{ID_INVESTIGACIONES}

consolidación de una ruta del vino, debidamente identificada que involucre las grandes y pequeñas bodegas, son algunos de los posibles proyectos mencionados.

En total se formulan diez pequeños itinerarios, la mayoría de ellos para ser recorridos en una jornada. Si bien se procura dotar a cada uno de una narración particular que guíe la visita, la propuesta queda abierta a modificaciones, incorporando o quitando establecimientos productivos y sitios de interés dependiendo de la actividad en los distintos momentos del año.

El trabajo identifica metas, plazos y acciones prioritarias dirigidas a la integración del desarrollo económico al desarrollo social y cultural de las comunidades anfitrionas y al mantenimiento y mejora de las calidades ambientales. Propone además el desarrollo de una promoción responsable que anuncie debidamente lo que el visitante encontrará en el área. Finalmente plantea la necesidad de fijar metas y plazos en escalas temporales variadas para comenzar a avanzar hacia el horizonte final en pasos acotados y posibles.

Esta propuesta finaliza en mayo de 2008 y unos meses después comienzan a instrumentarse, con apoyo de la Oficina de Turismo del gobierno departamental de Canelones, los primeros itinerarios propuestos en la misma.

\section{CONSIDERACIONES FINALES}

Si bien actualmente han empezado a desarrollarse en Uruguay nuevas propuestas basadas en esta visión de valorización del patrimonio natural y cultural y de la posibilidad de mejorar la calidad de vida de las comunidades locales, las tres propuestas presentadas, coincidentes sobre un mismo territorio, permiten dar cuenta de la evolución de esta manera de operar. 


\section{ID_INVESTIGACIONES}

Desde el primer antecedente en 1997, en que se propone el Programa de Turismo, Patrimonio y Ambiente del Área Suroeste y su concreción en el 2000, hasta la puesta en marcha de la propuesta para el Oeste canario en 2009, cada una ha constituido un antecedente de la anterior en un proceso de avance conceptual y metodológico. ¿Cuáles han sido los aportes concretos en cada caso? ¿Cuáles las dificultades para su concreción?

En el primer caso se integran patrimonio natural y cultural en un plano de igualdad en una propuesta de ordenamiento en una amplia región del territorio nacional, a la vez que se explicita la necesaria puesta en valor económico de ambos, como manera de fortalecerlos y defenderlos. Sin embargo la propuesta no involucra el patrimonio intangible y esto se debe seguramente a la manera en que fue gestada. Si bien el trabajo es llevado a cabo por encargo de los gobiernos departamentales y elaborado por un equipo interdisciplinar, este equipo trabaja desde la órbita del Ministerio. Es, por tanto, externo al territorio concreto. Aunque se realizan consultas y entrevistas con los actores locales, no se logra involucrar a los mismos en el proceso de elaboración.

Una restricción diferente en este caso proviene del ámbito políticoadministrativo ya que los tres departamentos que participan en el Parque Turístico responden a las tres fuerzas políticas predominantes en el momento de realizar la propuesta. Este aspecto imposibilita la coordinación de acciones sobre el territorio. El trabajo es entregado a las tres intendencias una vez finalizado. Cada una de ellas lo valora positivamente, sin embargo no realiza ninguna gestión para su concreción.

La segunda propuesta avanza sobre la anterior, profundizando en la definición de que se entiende por patrimonio en este enfoque, incorporando especialmente el concepto de paisaje cultural y aspectos vinculados a la consideración de la construcción histórica del territorio. Incluye además la construcción de los argumentos o narraciones con los que recorrer el territorio 


\section{ID_INVESTIGACIONES}

específico del parque. Si bien se consulta a los actores locales, especialistas y técnicos fundamentalmente del ámbito departamental, el trabajo es el resultado de una investigación académica que deviene en un nuevo proyecto territorial. Por lo tanto su resultado queda en la órbita de la universidad. No obstante tiene dos repercusiones: por un lado la generación de una base de datos sistematizada, a modo de inventario de recursos naturales y culturales, inexistente hasta el momento, y por otro lado una lectura del territorio metropolitano desde la perspectiva de un posible desarrollo turístico. El trabajo es presentado en varias oportunidades y consultado por varios técnicos de las administraciones municipales involucradas y conforma un referente para algunas investigaciones en el ámbito académico.

En el tercer ejemplo se trabaja con el mismo marco conceptual. Esta vez la demanda es del gobierno departamental, que está dispuesto a poner en marcha una propuesta que permita activar territorios interiores a través del desarrollo turístico alternativo a la oferta de sol y playa que caracteriza el turismo en el departamento. Existen ya algunas iniciativas en esta dirección, aunque no todas con el mismo alcance. Allí se localizan varias de las grandes bodegas que participan de diversas ofertas de recorridos y rutas de degustación de su producción. Si bien no incorporan pequeños productores en esta oferta, traen visitantes al área, brindando la posibilidad de activar otras alternativas. Se localizan además algunas organizaciones no gubernamentales que trabajan con pequeños productores y con temas ambientales en el entorno del río Santa Lucía.

Si bien el grado de desarrollo de este territorio es diverso, existen antecedentes de iniciativas locales para desarrollar alternativas que permitan complementar la producción.

En este caso el proyecto se apoya en el conocimiento del área, pero se construye paso a paso con los actores locales, que asumen un papel proactivo 


\section{ID_INVESTIGACIONES}

en la formulación de la propuesta, en sucesivas reuniones en distintas zonas del oeste de Canelones, reconociendo la heterogeneidad de situaciones y el sentido de pertenencia de los habitantes de un territorio que, a priori, parece homogéneo. El gobierno departamental apoya la gestión de alguno de los itinerarios formulados y logra llevarlos adelante, incorporándolos a la agenda turística del departamento. Esta es la única propuesta que logra algún grado de concreción inmediata.

De estas experiencias se desprenden cinco consideraciones a modo de lecciones aprendidas en este proceso.

La primera de ellas alude a la necesaria incorporación de múltiples escalas territoriales en el proceso de armado de la propuesta. Contextualizarla en un ámbito territorial mayor permite articularla con proyectos territoriales más amplios, generando sinergias o complementariedades, mientras que la pequeña escala permite desarrollar proyectos concretos fácilmente materializables, dando señales del avance de la propuesta. Este aspecto adquiere particular importancia en el territorio uruguayo, en el que las áreas de concentración de recursos pueden encontrarse separadas entre sí y alejadas de las zonas de concentración de población, posibles puertas de entrada al territorio. Visualizar la diversidad seguramente posibilitará multiplicar oportunidades de desarrollo.

La segunda se refiere al manejo del concepto de patrimonio en un sentido amplio e inclusivo de los componentes naturales y culturales, tangibles e intangibles y fundamentalmente considerar el patrimonio de escala local, como pieza fundamental de la propuesta. En este aspecto se ha producido un avance muy significativo en el ámbito nacional y las tres propuestas presentadas dan cuenta de ello. A modo de ejemplo vale la pena mencionar que en 1996 la Dirección Nacional de Ordenamiento Territorial del Ministerio de Vivienda, Ordenamiento Territorial y Medio Ambiente convocó a institutos de investiga- 


\section{ID_INVESTIGACIONES}

ción, oficinas técnicas del sector privado y público y organizaciones no gubernamentales a unas jornadas de exposición de temas de ordenamiento territorial. A la misma se presentaron 30 trabajos, de los cuales 16 fueron seleccionados por su mayor interés y actualidad. Estos describían, a criterio del Ministerio, los diferentes intereses y propuestas frente a los rápidos cambios que estaban operando en la sociedad. Los temas que aparecen en los trabajos seleccionados refieren a políticas de vivienda, al interés por el fortalecimiento de oficinas de planeamiento departamentales, cartas temáticas sobre usos del suelo, propuestas de preservación de áreas de valores ecológicos y a la identificación de actores y agentes de desarrollo local. En ninguno de ellos se plantea la gestión de los recursos patrimoniales como oportunidad para el desarrollo y no se menciona la vinculación de territorio y patrimonio (Medina 2005).

La tercera consideración se desprende directamente de la anterior. La consideración del patrimonio en un sentido amplio e inclusivo pone de manifiesto la necesidad de un enfoque interdisciplinario, que permita armar propuestas integrales, sustentables y sólidamente fundamentadas. La valorización del patrimonio natural, arqueológico, arquitectónico, artístico, así como los aspectos intangibles del mismo, requieren de conocimientos específicos que permitan no sólo una sólida fundamentación de sus valores, sino una adecuada gestión que haga posible mantener el equilibrio entre los visitantes y las comunidades anfitrionas.

En cuarto lugar, a través de las experiencias relatadas queda en evidencia la importancia de la participación de las comunidades locales en la formulación del proyecto y en la concreción del mismo. El rol de los gobiernos departamentales y el gobierno nacional es garantizar los ámbitos para que pueda darse la articulación de intereses, el apoyo al desarrollo de propuestas y a la capacitación de los actores locales. 


\section{ID_INVESTIGACIONES}

En quinto lugar, estas experiencias evidencian la pertinencia de trabajar con la tríada turismo-patrimonio-ambiente integrada a políticas de desarrollo en general y en particular de los gobiernos departamentales. La consideración simultánea de estos tres componentes permite fortalecer las acciones en cada uno de ellos a la vez que contribuye al progreso sustentable del territorio.

Finalmente el conocimiento y la difusión de los recursos patrimoniales han conformado el primer paso para desencadenar procesos de desarrollo basados en su puesta en valor. Los tres casos analizados demuestran que este aspecto es una constante, no sólo en la fase inicial de estos procesos, sino que se constituyen en actividades siempre presentes en los territorios en los que se desarrollaron estas propuestas.

\section{BIBLIOGRAFÍA}

CALA MATIZ, Bibiana (2001). La institución social del turismo vista desde la antropología I Congreso Virtual de Turismo Cultural, I Encuentro Regional de Turismo Cultural Disponible en www.naya.org.ar (20-02-2005)

CÓCERES Claudia, NOEL Gabriel, GÓMEZ Silvia, BIONDI Eduardo (2003). Recomendaciones para construir políticas para el turismo cultural, El turismo: espacio de diálogo intercultural; II Congreso Internacional de Turismo Cultural NayA, Disponible en www.naya.org.ar (20-02-2005)

CORBOZ, André (1983). El territorio como palimsesto en Martín Ramos Angel (de). Lo urbano en 20 autores contemporáneos, Barcelona, UPC/ETSAB, p.: 25-35.ISBN: 84-8301$752-0$

MEDINA Mercedes (2005). Construir el futuro revalorizando el pasado. El turismo cultural, una oportunidad de desarrollo en el territorio metropolitano de Montevideo. Tesis de la Maestría de Ordenamiento Territorial y Desarrollo Urbano de la Facultad de Arquitectura, en edición en Cuadernos del Territorio. 
ID_INVESTIGACIONES

MEDINA Mercedes, NUDELMAN Jorge (2008). Turismo agroproductivo en el oeste canario. Líneas estratégicas para su promoción”, Montevideo, CEEC-OPP-BID. ISBN 978/9974/8132/0/5.

MVOTMA, IMSJ, IMC, IMS (2009): Programa Integrado de Turismo, Patrimonio y Ambiente para el Área Suroeste, Cuadernos del Territorio No 3, Montevideo. ISBN 978-9974-7610-63

OMT (2010): Barómetro OMT del Turismo Mundial. Vol 8 №3. ISSN 1728-9254, disponible en www.unwto.org. (24-09- 2011)

OMT (1999): Guía para Administraciones Locales: Desarrollo Turístico Sostenible, Madrid, España ISBN 92-844-0307-3.

PEDERSEN Arthur (2002): Gestión del turismo en Sitios del Patrimonio Mundial: Manual práctico para administradores de sitios del Patrimonio Mundial. En www.unesdoc.unesco.org (24-09-2011)

Proyecto MVOTMA-DINAMA-PNUD-GEF (URU-06-G34), disponible en www.snap.org (2803-2008).

SABATÉ Joaquín (2003): Paisajes Culturales. El patrimonio como recurso básico para un nuevo modelo de desarrollo.Revista Urban $\mathrm{N}^{\circ} 6$ Madrid.

SABATÉ Joaquín, Lista Antoni (2001): Casos d'estudi europeus en Projectant l'eix del Llobregat. Paisatge cultural i desenvolupament regional, Barcelona, UPC/MIT, p.:78-190. ISBN 7653-793-X

www.snap.gub.uy, (5-05-2010), www.mintur.gub.uy, (20-02-2008). 


\title{
ates
}

A. 5

-31 in $42015)$ and

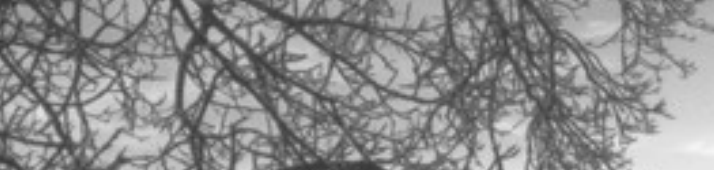
$1 \quad$ 1
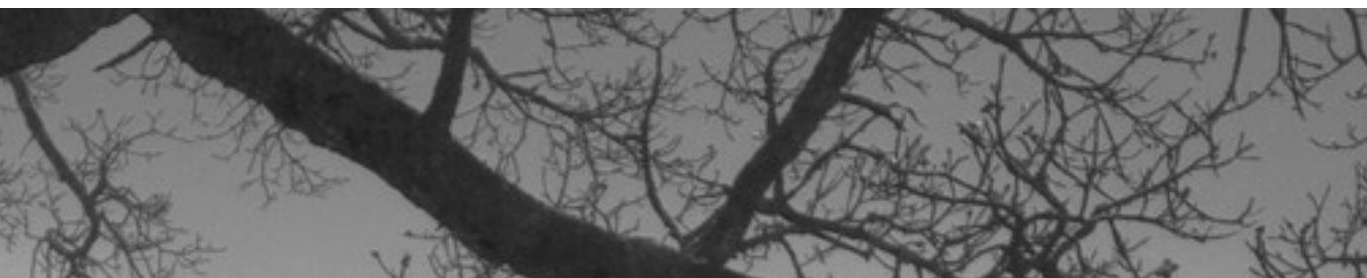
$1+$

\begin{abstract}
.
\end{abstract}
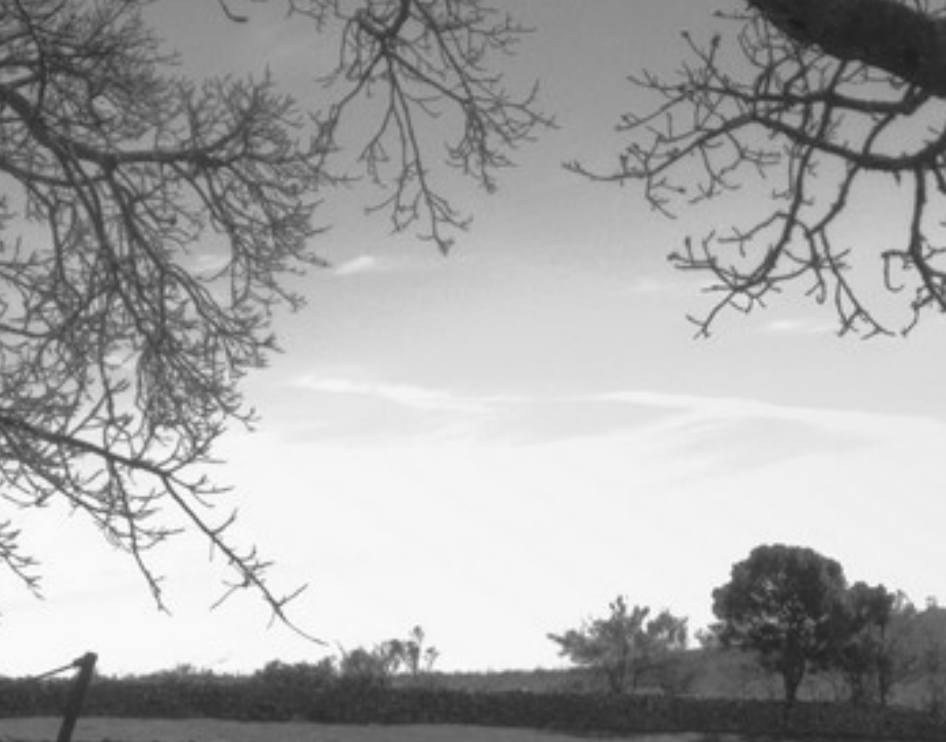